\section{Crystalline Diblock Conjugated Copolymers: Synthesis, Self-Assembly, and Microphase Separation of Poly(3-butylthiophene)- $b$-poly(3-octylthiophene)}

\author{
Pei-Tzu Wu, ${ }^{\dagger}$ Guoqiang Ren, ${ }^{\dagger}$ Chaoxu Li, ${ }^{\dagger}$ \\ Raffaele Mezzenga, ${ }^{*,+,}$ and Samson A. Jenekhe ${ }^{*, \dagger}$
}

Department of Chemical Engineering and Department of Chemistry, University of Washington, Seattle, Washington 98195-1750; Department of Physics and Fribourg Center for Nanomaterials, Fribourg University, Fribourg, Switzerland; and Néstlé Research Center, Vers-Chez-Les-Blanc, 1000 Lausanne 26, Switzerland

Conjugated polymer systems, including homopolymers, ${ }^{1}$ alternating/random copolymers, ${ }^{2}$ blends, ${ }^{3}$ and block copolymers, ${ }^{4,5}$ as semiconductors for electronic and optoelectronic applications are of continuing great interest. ${ }^{6,7}$ In general, multicomponent conjugated polymer systems such as blends and block copolymers offer the opportunity to optimize and tailor electronic and optical properties while also having the potential to observe novel phenomena (e.g., energy transfer, charge transfer) not feasible in homopolymers and random/alternating copolymers. ${ }^{5}$ Compared to blends, ${ }^{3}$ block copolymers are of special interest because of their superior self-assembly features and the improved control of the nanoscale domain sizes of their assembled structures. Indeed, the synthesis, self-assembly, and properties of rod-coil block copolymers, having a $\pi$-conjugated (rodlike) block and a coillike nonconjugated block, have been extensively studied. ${ }^{4}$ Although experimental examples of allconjugated block copolymers have been known since $1996,{ }^{5 a}$ their synthesis, solution-phase self-assembly, melt-phase selfassembly, and properties remain to be fully investigated. ${ }^{5,8}$

Recently, block copolythiophenes with crystalline-amorphous diblock architecture incorporating a crystalline poly(3-hexylthiophene) (P3HT) block were successfully synthesized by quasiliving chain growth polymerization, including poly\{3-[2-(2methoxyethoxy)ethoxy]methylthiophene $\},{ }^{8 \mathrm{~b}}$ poly[3-(2ethylhexylthiophene)], ${ }^{8 \mathrm{c}}$ or poly(3-phenoxymethylthiophene $)^{8 \mathrm{~d}}$ as the amorphous segment. The thin-film morphology of these crystalline-amorphous diblock copolythiophenes was shown by atomic force microscopy (AFM) to be microphase-separated into crystalline and amorphous domains. For many electronic and optoelectronic applications such as field-effect transistors and photovoltaic devices, where high carrier mobilities and high absorption coefficients are important, ${ }^{6,7}$ amorphous domains are undesirable. We report herein the synthesis and self-assembly of crystalline-crystalline diblock copoly(3-alkylthiophene)s. Two compositions of the new regioregular poly(3-butylthiophene)- $b$-poly(3-octylthiophene) (P3BT- $b$-P3OT) were found to self-assemble into crystalline nanowires in solution and shown by wide-angle X-ray scattering (WAXS) and small-angle X-ray scattering (SAXS) to be microphase-separated from the melt

* Authors for all correspondence. E-mail: raffaele.mezzenga@unifr.ch (R.M.); jenekhe@u.washington.edu (S.A.J.)

University of Washington.

Fribourg University.

Nestlé Research Center. phase into two distinct crystalline domains with a lamellar structure.

The synthesis of the diblock copoly(3-alkylthiophene)s was carried out by a modified Grignard metathesis method (GRIM) ${ }^{1 \mathrm{~b}, 8}$ as illustrated in Scheme 1 . The P3OT block was first synthesized by polymerization of 2,5-dibromo-3-octylthiophene, followed by the addition of activated 2,5-dibromo-3butylthiophene monomer solution, giving the diblock copolymer system, poly(3-butylthiophene)- $b$-poly(3-octylthiophene). Two compositions, denoted $\mathrm{BO} 50$ and $\mathrm{BO} 76$, were synthesized by using the feed ratios of 2,5-dibromo-3-octylthiophene to 2,5dibromo-3-butylthiophene of $1: 1$ and $1: 2$, respectively. The actual compositions of BO50 and BO76 were determined from the ${ }^{1} \mathrm{H}$ NMR spectra (Figure S1), based on the terminal methyl groups of the butyl and octyl side chains which showed resonances at 1.0 and $0.9 \mathrm{ppm}$, respectively. The regioregularity of both BO samples was determined to be $94 \%$ by using the resonance of the $\alpha$-methylene protons. ${ }^{\text {1a,b }}$ The number-average molecular weights $\left(M_{\mathrm{n}}\right)$ of BO50 and BO76 were 17300 and $11400 \mathrm{~g} / \mathrm{mol}$, with a polydispersity index of 1.69 and 1.60 , respectively, by gel permeation chromatography (GPC) analysis. The large polydispersity indices are a result of the poor selectivity of activation by isopropylmagnesium chloride of 2,5dibromo-3-octylthiophene and 2,5-dibromo-3-butylthiophene. The polydispersity index could be potentially reduced by using the asymmetric 2-bromo-5-iodo-3-alkylthiophene as the starting monomer. ${ }^{8}$

Figure 1a shows the differential scanning calorimetry (DSC) scans of BO50 and BO76. Two melting transitions with endothermic peaks at 246 and $190{ }^{\circ} \mathrm{C}$ and two corresponding exothermic recrystallization peaks at 204 and $148{ }^{\circ} \mathrm{C}$ were observed in the DSC scan of BO50. The observed two melting transitions in BO50 are characteristic of its diblock architecture composed of P3BT and P3OT blocks, which have melting transitions at 243 and $190{ }^{\circ} \mathrm{C}$, respectively, as homopolymers. ${ }^{9}$ The slight variation in the melting transition values of BO50 (246 and $190^{\circ} \mathrm{C}$ ) compared to the homopolymers of P3BT and P3OT is likely due to the different molecular weights of the homopolymers. ${ }^{9}$ In the case of BO76, only one distinct melting transition peak $\left(263{ }^{\circ} \mathrm{C}\right)$ with a recrystallization peak at $223{ }^{\circ} \mathrm{C}$ was observed and is clearly due to the P3BT block. The absence of the melting transition of the P3OT block in BO76 may be due to its low degree of polymerization within the crystalline-crystalline diblock copolymer.

The photophysical properties of the regioregular diblock copoly(3-alkylthiophene)s were investigated by optical absorption and photoluminescence (PL) spectroscopies. In chloroform solution, both BO50 and BO76 had identical absorption maxima

Scheme 1. Synthesis of Poly(3-butylthiophene)-b-poly(3-octylthiophene)

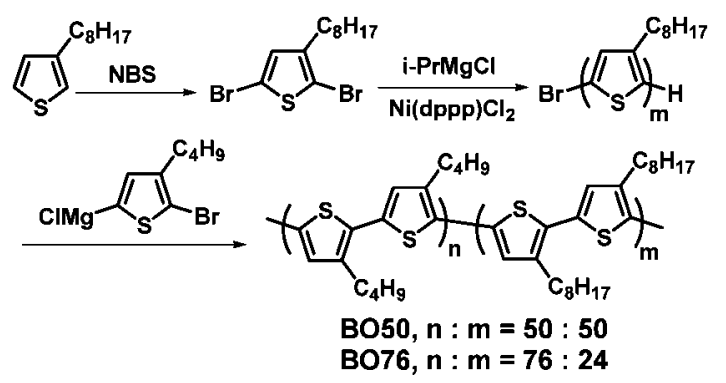



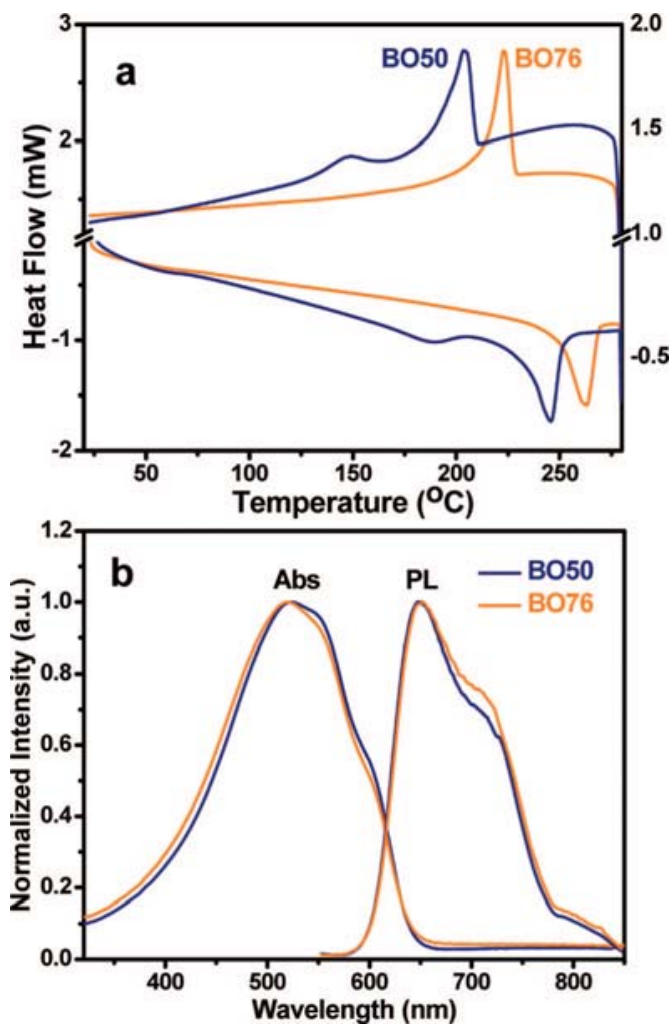

Figure 1. (a) DSC scans of poly(3-butylthiophene)-b-poly(3-octylthiophene), BO50 and BO76. (b) Absorption and photoluminescence spectra of BO50 and BO76 as thin films.

at $453 \mathrm{~nm}$ and identical PL emission maxima at $576 \mathrm{~nm}$ (Figure $\mathrm{S} 2$ ), as expected since they share the same polythiophene main chain. ${ }^{1 \mathrm{a}}$ The optical absorption spectra of thin films of BO50 and BO76 (Figure 1b) with absorption maxima of 520-525 $\mathrm{nm}$ and shoulder peaks at $550-600 \mathrm{~nm}$ are very similar to the related $\mathrm{P} 3 \mathrm{BT}$ and $\mathrm{P} 3 \mathrm{OT}$ homopolymers. BO50 and $\mathrm{BO} 76$ have PL emission maxima at $651-653 \mathrm{~nm}$ with the same shoulder peak at $710 \mathrm{~nm}$ (Figure 1b). We note that the long-wavelength shoulder peak in the absorption spectra of BO50 and BO76 films is associated with crystallinity, resulting from strong intermolecular interactions among regioregular poly(3-alkylthiophene) chains. ${ }^{1}$

We investigated the self-assembly of the diblock copoly(3alkylthiophene)s in solution, focusing on the nature of aggregates formed in solution by spontaneous self-assembly. A bulk solution of BO50 in 1,2-dichlorobenzene (ODCB) $(10 \mathrm{mg} / \mathrm{mL})$ was prepared at room temperature, filtered (through a $0.45 \mu \mathrm{m}$ filter), and stored for $24 \mathrm{~h}$. The resulting dispersion was spincoated onto a silicon wafer for AFM imaging or was diluted 10 times with ODCB and drop-casted onto a TEM grid for TEM imaging. Figure 2a shows the AFM height image of the BO50 thin film obtained from the dispersion, revealing nanowires (NWs) throughout the surface. TEM images of the BO50 dispersion similarly revealed NWs with a relatively narrow width $(13.5 \mathrm{~nm})$ and broad length distribution $(250-1000 \mathrm{~nm})$, as exemplified in Figure 2b. BO76 solutions in ODCB were observed to similarly form BO76 NWs with an average width of $13.6 \mathrm{~nm}$ and aspect ratios of 15 to 51 (Figures S3 and S4). It is interesting to contrast the assembly of NWs with micelles, vesicles, and similar aggregates typically formed by conventional block copolymers, including those containing conjugated polymers. ${ }^{4 a}$ The observed solution phase self-assembly of P3BT$b$-P3OT into nanowires has important implications for understanding the assembly of block copolythiophenes and for optoelectronic applications. Although the detailed molecular packing within the diblock copolymer NWs remains to be investigated, the fact that the assembly of P3BT- $b$-P3OT NWs occurs in solution very similar to the assembly of NWs from $\mathrm{P}_{3 \mathrm{BT}}{ }^{7}$ and $\mathrm{P} \mathrm{OT}^{10}$ homopolymers means that $\pi$-stacking interactions are dominant relative to interactions of the side chains. Similar to the poly(3-alkylthiophene) homopolymer NWs, we expect the block copolythiophene NWs to be useful in bulk heterojunction type photovoltaic devices by virtue of their high-mobility transport of holes among the network of crystalline NWs and advantageous nanoscale morphology in blends with fullerenes. ${ }^{7}$

Figure 2c shows wide-angle X-ray diffraction (WAXD) scans on the drop-cast films from dispersions of BO50 and BO76 NWs in 1,2-dichlorobenzene. BO50 NWs are clearly crystalline, showing two strong diffraction peaks at $2 \theta$ angles of $4.51^{\circ}$ and $6.34^{\circ}$, corresponding to two $d_{100}$ values of 19.6 and $13.9 \AA$, respectively. The $19.6 \AA$ spacing is due to the interlayer stacking distance between P3OT blocks in a layered packing structure $\left(d_{\text {Р3ОT }}\right)$, whereas the $13.9 \AA$ spacing comes from the interlayer stacking distance between P3BT blocks, $d_{\mathrm{P} 3 \mathrm{BT}}$. The peaks at $8.71^{\circ}$ and $12.86^{\circ}$ with lower intensities are associated with the (200) diffraction from P3OT and P3BT blocks in BO50, respectively. The $(100)_{\mathrm{P} 3 \mathrm{BT}}$ and (100) Р3От $_{\text {diffraction showed }}$ comparable intensity due to the 50:50 composition of P3BT and P3OT blocks in BO50. In the case of BO76 NWs, the peak maximum at $6.91^{\circ}$ and a less intense peak at $5.00^{\circ}$ are due to two $d_{100}$ values of $12.8\left(d_{\mathrm{P} 3 \mathrm{BT}}\right)$ and $17.7 \AA\left(d_{\mathrm{P} 3 \mathrm{OT}}\right)$, respectively. We note that the observed $d_{\text {Р3От }}$ values $(17.7-19.6 \AA)$ seen in the BO50 and BO76 NWs are smaller than the reported $d_{100}$ value (20.1 $\AA$ ) for the P3OT homopolymer, ${ }^{\text {a }}$ suggesting partial interdigitation between the side chains and/or the occurrence of tilting of the octyl chains in P3BT- $b$-P3OT. On the other hand, BO50 and BO76 have a $d_{\mathrm{P} 3 \mathrm{BT}}$ in the range of 12.8-13.9 $\AA$, which is very close to the $d_{100}$ of $12.63 \AA$ reported for the P3BT homopolymer. ${ }^{1 \text { a }}$ The P3BT- $b$-P3OT copolymers clearly showed two distinct crystalline domains with relative intensities of WAXD reflection peaks from the P3BT- and P3OT-rich domains approximately scaling with the composition ratio ( $n$ : $m$ ); BO50 had two comparable reflection peaks, whereas BO76 exhibits a major reflection peak due to the dominant P3BT phase with a smaller peak due to P3OT (Figure 2c). These WAXD results show that $\mathrm{BO} 50$ and $\mathrm{BO} 76 \mathrm{NWs}$ are crystalline with two distinct $d_{100}$ values, $d_{\mathrm{P} 3 \mathrm{BT}}$ and $d_{\mathrm{P} 3 \mathrm{OT}}$, and suggest microphase separation among P3BT- and P3OT-rich domains.

To investigate the melt-phase behavior and solid-state morphology of BO50 and BO76, films spin-casted from chloroform solutions and annealed at $280{ }^{\circ} \mathrm{C}$ under high vacuum $\left(10^{-7}\right.$ mbar) for $12 \mathrm{~h}$ were characterized by wide-angle X-ray scattering (WAXS) and small-angle X-ray scattering (SAXS). Figure $3 \mathrm{a}$ shows the WAXS spectra of the two diblock copolymers. In BO50, two scattering vectors in the low- $q$ region $\left(q=3.18\right.$ and $\left.4.76 \mathrm{~nm}^{-1}\right)$ are seen and can be identified as corresponding to real-space $d$ values of 19.7 and $13.2 \AA$, respectively. The smaller $d$ value (13.2 $\AA$ ) in BO50 is clearly due to the (100) reflection associated with the interlayer spacing of the P3BT segments, whereas the larger $d$ value (19.7 $\AA$ ) originates from the (100) reflection from the interlayer spacing of the P3OT segments. These results mean that two crystalline domains are formed by the regioregular P3BT and P3OT blocks in the diblock copolymers. In the high- $q$ region, together with a broad halo resulting from the amorphous side chains, the peak at $16.5 \mathrm{~nm}^{-1}$ is associated with the $(020)$ reflection, which corresponds to a $\pi-\pi$ stacking distance of $3.8 \AA$ and is the 

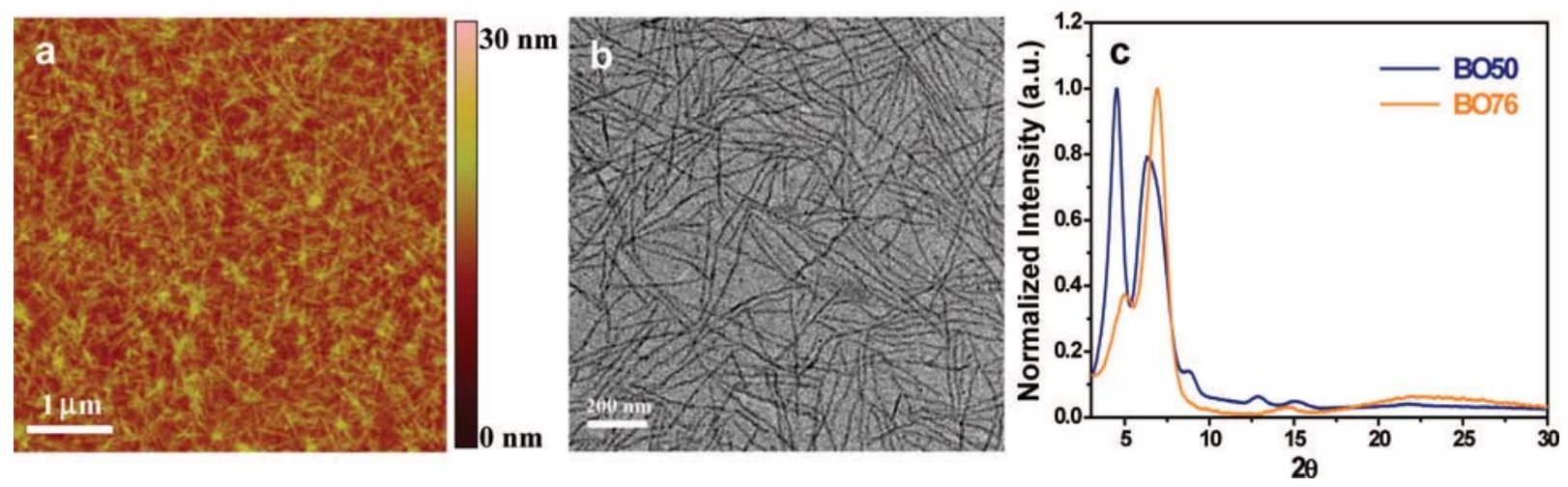

Figure 2. AFM height image (a) and TEM image (b) of BO50 nanowires assembled from a $10 \mathrm{mg} / \mathrm{mL}$ solution. (c) WAXD spectra of BO50 and BO76 as drop-cast films, dried at room temperature.
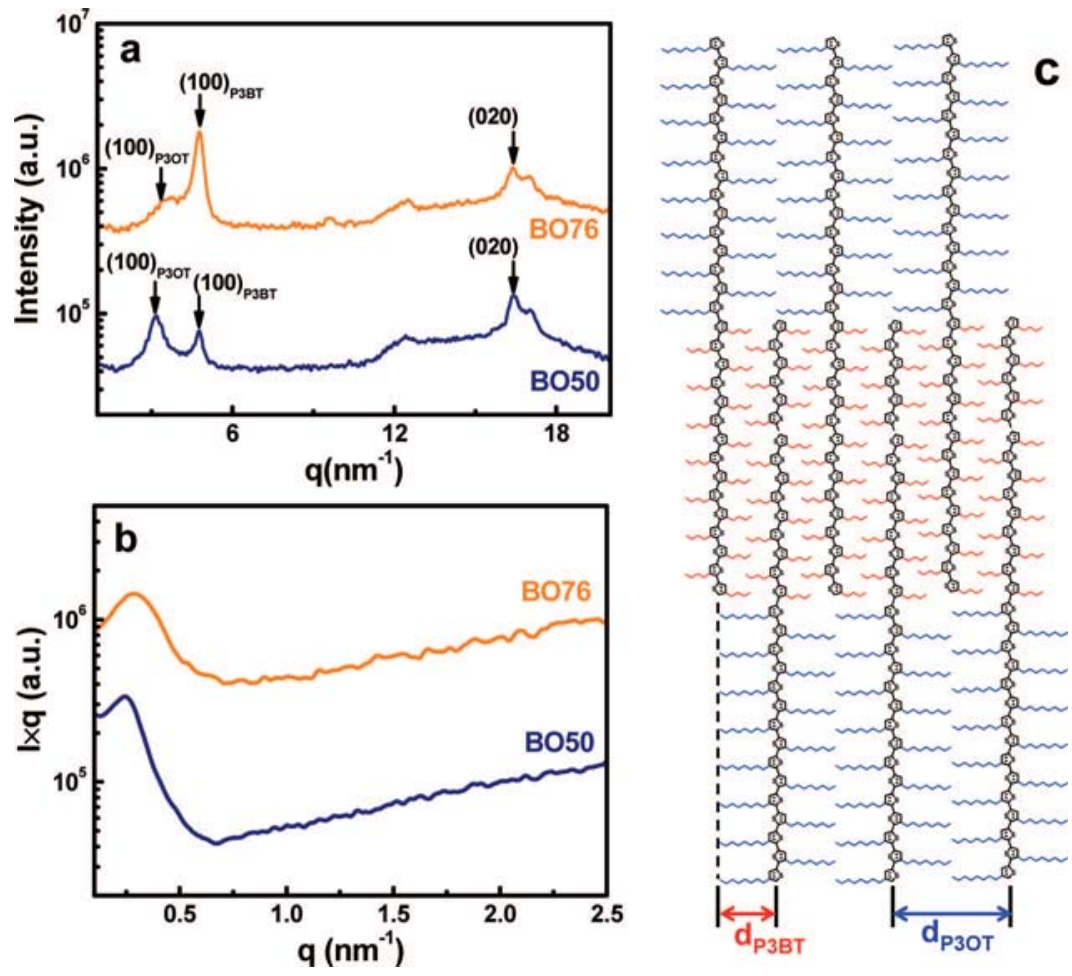

Figure 3. WAXS spectra (a) and SAXS spectra (b) of BO50 and BO76 films annealed at $280{ }^{\circ} \mathrm{C}$ in vacuum. (c) Schematic model of microphaseseparated lamellar structure of P3BT- $b$-P3OT with two interlayer spacings $\left(d_{\mathrm{P} 3 \mathrm{BT}}\right.$ and $\left.d_{\mathrm{P} 3 \mathrm{OT}}\right)$ observed in WAXS.

same as the reported $\pi-\pi$ stacking distance in P3BT and P3OT. ${ }^{9 a}$ Similarly, two scattering vectors $(q)$ at 3.37 and 4.77 $\mathrm{nm}^{-1}$, corresponding to two $d$ spacings of 18.6 and $13.2 \AA$, respectively, were observed in the case of BO76. These results confirmed the existence of two different crystalline domains formed by segregated P3BT and P3OT blocks in both BO50 and BO76. Whereas the DSC scan of BO76 could not resolve the two melting transitions due to P3BT and P3OT blocks, the WAXS spectrum of BO76 shows a strong (100) Рзвт peak along with a shoulder $(100)_{\text {Рзот }}$ peak, clearly resolving the two crystalline domains. In the case of BO50, the crystalline nature of two separated P3BT and P3OT domains can be identified from both DSC and WAXS scans.

The SAXS spectra of BO50 and BO76 films, annealed at $280^{\circ} \mathrm{C}$ as with the WAXS samples in Figure $3 \mathrm{a}$, are shown in Figure $3 \mathrm{~b}$. A distinct reflection peak at a scattering vector of $0.25 \mathrm{~nm}^{-1}$ is observed in the BO50 diblock copolymer, showing that there is microphase separation with a domain spacing of $25.7 \mathrm{~nm}$. Similarly, the low- $q$ reflection peak $\left(q=0.29 \mathrm{~nm}^{-1}\right)$ observed in the BO76 sample means that there is microphase separation with a domain spacing of $21.8 \mathrm{~nm}$. Taken together with the previously discussed WAXS results, and considering the highly rigid-rod-like nature of the block copolymer considered, these SAXS results suggest a melt-phase assembly of the diblock copoly(3-alkylthiophene)s into a microphase-separated lamellar structure with two crystalline domains characteristic of the two different side chains (Figure 3c). Because the lamellar period measured by SAXS is consistently smaller than the corresponding contour lengths of the block copolymers estimated from their molecular weights $(25.7 \mathrm{~nm}$ vs $40 \mathrm{~nm}$ and $21.8 \mathrm{~nm}$ vs $28.7 \mathrm{~nm}$ for BO50 and BO76, respectively), a lamellar structure of smectic $\mathrm{C}$ nature, with tilt angles of rods with respect to the lamellar interface is to be expected, although the large polydispersity of the present systems does not allow precisely identifying the tilt angles. Smectic C lamellar phases were previously reported by the authors for diblocks and triblock copolymers containing $\pi$-conjugated blocks. ${ }^{11}$

In conclusion, we have synthesized two crystalline-crystalline diblock copoly(3-alkylthiophene)s and investigated their selfassembly in solution and in the melt phase. Both compositions 
of poly(3-butylthiophene)- $b$-poly(3-octylthiophene) (BO50 and BO76) in solution were found to self-assemble into crystalline nanowires with widths of 13.5-13.6 nm and aspect ratios of 15-74, as characterized by AFM and TEM. WAXS and SAXS studies confirmed that these diblock copolymers in the melt phase assemble into a microphase-separated lamellar structure with two crystalline domains (length scale $\sim 22-26 \mathrm{~nm}$ ) reflecting the two different side chains. Our results suggest that all-conjugated block copolymers with crystalline-crystalline diblock architecture are promising systems for further studies in the nature of block copolymer self-assembly and as nanostructured polymer semiconductors for electronic and optoelectronic applications.

Acknowledgment. Our work was supported by the Department of Energy, Basic Energy Sciences (DE-FG02-07ER46467), the NSF (DMR-0805259 and DMR-0120967), and in part by the AFOSR EHSS-MURI (FA9550-06-1-0326). Part of this work was conducted at the University of Washington NanoTech User Facility, a member of the NSF National Nanotechnology Infrastructure Network (NNIN). C.L. and R.M. thank the Swiss National Science Foundation for financial support.

\section{References and Notes}

(1) (a) Chen, T.-A.; Wu, X.; Rieke, R. D. J. Am. Chem. Soc. 1995, 117, 233-244. (b) McCullough, R. D.; Lowe, R. D.; Jayaraman, M.; Anderson, D. L. J. Org. Chem. 1993, 58, 904-912. (c) Sirringhaus, H.; Brown, P. J.; Friend, R. H.; Nielsen, M. M.; Bechgaard, K. W.; Langeveld-Voss, B. M.; Spiering, A. J. H.; Janssen, R. A. J.; Meijer, E. W.; Herwig, P.; de Leeuw, D. M. Nature (London) 1999, 401, 685688. (d) Mena-Osteritz, E.; Meyer, A.; Langeveld-Voss, B. M. W.; Janssen, R. A. J.; Meijer, E. W.; Bäuerle, P. Agnew. Chem., Int. Ed. 2000, 39, 2680-2684.

(2) (a) Zhu, Y.; Champion, R. D.; Jenekhe, S. A. Macromolecules 2006, 39, 8712-8719. (b) Zen, A.; Saphiannikova, M.; Neher, D.; Asawapirom, U.; Scherf, U. Chem. Mater. 2005, 17, 781-786. (c) Yamamoto, T.; Koizumi, T. Polymer 2007, 48, 5449-5472. (d) Leclère, Ph.; Surin, M.; Brocorens, P.; Cavallini, M.; Biscarini, F.; Lazzaroni, R. Mater.
Sci. Eng. R 2006, 55, 1-56. (e) Reddinger, J. L.; Reynolds, J. R. Adv. Polym. Sci. 1999, 145, 57-122.

(3) (a) Babel, A.; Jenekhe, S. A. J. Phys. Chem. B 2003, 107, 17491754. (b) Alam, M. M.; Tonzola, C. J.; Jenekhe, S. A. Macromolecules 2003, 36, 6577-6587.

(4) (a) Jenekhe, S. A.; Chen, X. L. Science 1998, 279, 1903-1907. (b) Chen, X. L.; Jenekhe, S. A. Science 1999, 283, 372-375. (c) Chen, X. L.; Jenekhe, S. A. Macromolecules 2000, 33, 4610-4612. (d) Liu, J.; Sheina, E.; Kowalewski, T.; McCullough, R. D. Angew. Chem., Int. Ed. 2002, 41, 329-332. (e) Leclère, Ph.; Calderone, A.; Marsitzky, D.; Francke, V.; Geerts, Y.; Müllen, K.; Bredas, J. L.; Lazzaroni, R. Adv. Mater. 2000, 12, 1042-1046. (f) Stalmach, U.; de Boer, B.; Videlot, C.; van Hutten, P. F.; Hadziioannou, G. J. Am. Chem. Soc. 2000, 122, 5464-5472. (g) Olsen, B. D.; Segalman, R. A. Macromolecules 2005, 38, 10127-10137. (h) Sary, N.; Rubatat, L.; Brochon, C.; Hadziioannou, G.; Ruokolainen, J.; Mezzenga, R. Macromolecules 2007, 40, 6990-6997. (i) Sary, N.; Brochon, C.; Hadziioannou, G.; Mezzenga, R. Eur. Phys. J. E 2007, 24, 379-384.

(5) (a) Chen, X. L.; Jenekhe, S. A. Macromolecules 1996, 29, 6189-6192. (b) Scherf, U.; Gutacker, A.; Koenen, N. Acc. Chem. Res. 2008, 41, 1086-1097. (c) Liang, Y.; Wang, H.; Yuan, S.; Lee, Y.; Gan, L.; Yu, L. J. Mater. Chem. 2007, 17, 2183-2194. (d) Plank, H.; Güntner, R.; Scherf, U.; List, E. J. W. Adv. Funct. Mater. 2007, 17, 1093-1105. (e) Tu, G.; Li, H.; Forster, M.; Heiderhoff, R.; Balk, L. J.; Scherf, U. Macromolecules 2006, 39, 4327-4331.

(6) (a) Zaumseil, J.; Sirringhaus, H. Chem. Rev. 2007, 107, 1296-1323. (b) Günes, S.; Neugebauer, H.; Sariciftci, N. S. Chem. Rev. 2007, 107, 1324-1338. (c) Yang, X.; Loos, J. Macromolecules 2007, 40, 1353-1362. (d) Coakley, K. M.; McGehee, M. D. Chem. Mater. 2004, 16, 4533-4542. (e) Alam, M. M.; Jenekhe, S. A. Chem. Mater. 2004, $16,4647-4656$.

(7) (a) Xin, H.; Kim, F. S.; Jenekhe, S. A. J. Am. Chem. Soc. 2008, 130, 5424-5425. (b) Xin, H.; Kim, F. S.; Ren, G.; Jenekhe, S. A. Chem. Mater. 2008, 20, 6199-6207.

(8) (a) Iovu, M. C.; Sheina, E. E.; Gil, R. R.; McCullough, R. D. Macromolecules 2005, 38, 8649-8656. (b) Yokozawa, T.; Adachi, I.; Miyakoshi, R.; Yokoyama, A. High Perform. Polym. 2007, 19, 684699. (c) Zhang, Y.; Tajima, K.; Hirota, K.; Hashimoto, K. J. Am. Chem. Soc. 2008, 130, 7812-7813. (d) Ohshimizu, K.; Ueda, M. Macromolecules 2008, 41, 5289-5294.

(9) (a) Causin, V.; Marega, C.; Marigo, A.; Valentini, L.; Kenny, J. M. Macromolecules 2005, 38, 409-415. (b) Malik, S.; Nandi, A. K. J. Polym. Sci., Polym. Phys. 2002, 40, 2073-2085.

(10) Samitsu, S.; Shimomura, T.; Heike, S.; Hashizume, T.; Ito, K. Macromolecules 2008, 41, 8000-8010.

(11) (a) Sary, N.; Mezzenga, R.; Brochon, C.; Hadziioannou, G.; Ruokolainen, J. Macromolecules 2007, 40, 3277-3286. (b) Rubatat, L.; Kong, X.; Jenekhe, S. A.; Ruokolainen, J.; Hojeij, M.; Mezzenga, R. Macromolecules 2008, 41, 1846-1852. 